\title{
Two product, two region production, inventory, and transportation problems
}

\author{
Jong Hyup Lee ${ }^{1}$, Jung Man Hong, ${ }^{2, *}$ \\ ${ }^{1}$ Dept. of Information and Communications Engineering, Inje University, Gimhae, Republic of Korea \\ ${ }^{2}$ LG CNS, Seoul, Republic of Korea (* Corresponding Author)
}

Email address:

icjhlee@inje.ac.kr (Jong Hyup Lee), hongjungman@gmail.com (Jung Man Hong)

\section{To cite this article:}

Jong Hyup Lee, Jung Man Hong. Two Product, Two Region Production, Inventory, and Transportation Problems. International Journal of Economics, Finance and Management Sciences. Vol. 2, No. 6, 2014, pp. 313-318. doi: 10.11648/j.ijefm.20140206.13

\begin{abstract}
A deterministic production and transportation planning problem is considered over a finite time horizon for two products that can be produced in each of two regions. Each region uses its own facility to supply the demands for two products. Demands for product 2 in one region can be satisfied either by its own production or by transportation from other region, while no transportation between two regions is allowed for product 1. Production, inventory and transportation costs are assumed to be non-decreasing and concave. The objective is to find the schedule of production and transportation in each region by which the total cost over the horizon is minimized. Using a network flow approach, we develop a dynamic programming algorithm that can find an optimal policy.
\end{abstract}

Keywords: Production Planning, Network Flow, Dynamic Programming

\section{Introduction}

Until the 1980s, traditional dynamic lot-sizing models have been applied for solving the production and inventory problems. Various production and inventory models have been developed for single and multi-facility problem with a finite planning horizon of $T$ periods. For example, a single product, single facility dynamic lot-sizing problem with time-dependent concave costs have been first proposed in [1]. In certain types of concave cost networks, the algorithm to determine minimum cost flows has been considered in [2]. A similar problem under the assumption that the production levels were restricted to period-dependent capacity limits has also studied in [3]. They have devised a dynamic programming and shortest path algorithm only for the case of constant capacity. A single-facility multi-product production planning problem with no capacity constraint was proposed in [4]. A deterministic capacity expansion model for two facility types has been described in [5]. At the beginning of each period, facility $i(i=1,2)$ could be expanded, either by new construction, or by conversion of idle capacity associated with facility $j(j=1,2$ and $j \neq i)$ to accommodate the demand for facility $i$. In these papers, they have assumed the cost functions to be concave, but they haven't considered the transportation of the products. Above these papers, a lot of papers have been published handling the problems related to the dynamic lot-sizing for many different types of production environment. [6-10] Whereas, in the supply chain management (SCM) area that developed in earnest in the 1990s [11], there have been many efforts to resolve the integrated problems considering key business processes of production, storage and distribution, from original suppliers through end users. [12-14] However, they have assumed the cost functions to be linear due to the intrinsic complexity of the concave function. But it is more realistic that the cost functions are assumed to be non-decreasing and concave reflecting the economies of scale. In this paper, based on the traditional dynamic lot-sizing problem, we consider integrated production, inventory and transportation problem. We assume that the cost functions used in this problem are to be non-decreasing and concave in order to represent the realistic cost functions. Though the problem suggested in this paper does not resolve the general integrated supply chain problem, it is meaningful in respect of applying more realistic cost functions to somewhat simplified supply chain problem. In this paper, we consider a production, inventory and transportation planning problem for which two production regions are involved. In each region, a single facility manufactures two items (or products) each taking a fixed part of the whole production amount to satisfy its own demands over the discrete $T$ periods. In the problem, we assume that the transportation is allowed only for product 2 from one region to another. 
Such a problem involving multi-products by a single facility occurs frequently in two functionally distinctive classes of manufacturing industries; one in chemical industry and the other in machinery (or food) industry. Each of these cases is described in a bit more detail. In the first case, the involved input resource contains various components in a fixed quantity proportion, which are distinctively converted into their corresponding commercial products in the fixed proportion. Therefore, all the products are distinctively various in both quality and quantity. As an example, an oil refinery problem can be considered, for which each unit of crude oil is refined to produce two different products, say gasoline and a fine chemical resource, in the fixed quantity ratios, say $\alpha_{1}>0$ and $\alpha_{2}>0$, respectively. As another example, consider a production system, for which each operation generates by-product in a fixed ratio, say $\alpha$. In the other class, the employed facility (plant) with a certain number of distinctive sub-operation lines attached operates to supply a resource (fixed) simultaneously to all the attached lines, each of which generates its own commercial products. Therefore, all the products are in the same quality but their quantities are distinctively dependent on the capacities (fixed) of the involved sub-operation lines. As an example, a steel processing system can be considered, for which each unit of steel is processed to produce nail and wire simultaneously in a pre-specified weight proportion.

In connection with transportation, the following examples can be taken into account. As the first example, an import policy for cotton is implemented at two regions, for which two kinds of blended yarns are manufactured in each region, one with $40 \%$ chemical fiber mixed and the other one with $60 \%$ chemical fiber mixed. Only the blended yarn with $60 \%$ chemical fiber mixed can be transported from one region to another. In the case, the chemical fiber resource is assumed to be plentifully available for blending the two distinctive yarns. As another example, consider a general chemical process, for which a by-product is generated and its transportation from one region to another is allowed for its own market demands.

The associated policy consists of determining the production and transportation amounts in each period at each region over the planning horizon. The incurring costs include both the production and transportation costs and the inventory holding costs. All the involving cost functions depend on period and are non-decreasing concave. The goal of this paper is then to find an optimal policy by which the total cost incurred over the whole horizon is minimized.

The remainder of this paper is organized as follows. In Section 2, the proposed problem is formulated as a non-linear programming problem. Then we show that the constraints in the formulated model can be described as a single source network problem. The characteristics of an optimal solution are identified and a dynamic programming approach is addressed to solve the problem in Section 3. In Section 4, the algorithm is illustrated with a numerical example. Finally, a conclusion is drawn in Section 5.

\section{Model Formulation}

The model assumes a finite planning horizon of $T$ periods in which each production (and each transportation, if necessary) occurs instantaneously and simultaneously at the beginning of each period. Useful notations are defined as follows:

$r_{i t}^{k}=$ Demand for product $i$ in region $k$ at period $t$, where $r_{i t}^{k} \mathrm{~s}$ are positive integers

$R_{i}^{k}(t)=\sum_{h=t}^{T} r_{i h}^{k}$

$x_{i t}^{k}=$ Amount of production for product $i$ in region $k$ at the beginning of period $t$

$I_{i t}^{k}=$ Amount of inventory for product $i$ in region $k$ at the end of period $t$

$y_{t}^{k}=$ Transportation amount of product 2 from region $k$ to region $j$ at the beginning of period $t$, where $k, j=1,2$ and $k \neq j$

$$
P_{i t}^{k}\left(x_{i t}^{k}\right)=\text { Cost of producing } x_{i t}^{k} \text { at the beginning of period }
$$

$t$

$Q_{t}^{k}\left(y_{t}^{k}\right)=$ Cost of transporting $y_{t}^{k}$ from region $k$ to region $j$ at the beginning of period $t$

$H_{i t}^{k}\left(I_{i t}^{k}\right)=$ Inventory holding cost of $I_{i t}^{k}$ from period $(t-1)$ to period $t$

It is assumed that $P_{i t}^{k}(\bullet), Q_{t}^{k}(\bullet)$, and $H_{i t}^{k}(\bullet)$ are non-decreasing concave functions. And also we assume that $x_{t}^{k}=x_{1 t}^{k}=x_{2 t}^{k}$. This assumption implies that one unit of resource consumption leads to all the involved products each resulting in its own single commodity unit. From the above assumption, we can let $P_{t}^{k}\left(x_{t}^{k}\right)=\sum_{i=1}^{2} P_{i t}^{k}\left(x_{i t}^{k}\right)$, for all $t$.

There is no loss of generality in assuming that both the initial and final inventories are zero for each of the products, since otherwise the original problem can be adjusted by employing certain fictitious periods or artificial demands at the end of period $T$.

The problem is then to minimize the total costs of production, inventory and transportation, which can be formulated as follows:

(PB)

$$
\begin{aligned}
\operatorname{minimize} F(z) & =\sum_{t=1}^{T}\left[\sum _ { k = 1 } ^ { 2 } \left[P_{t}^{k}\left(x_{t}^{k}\right)+Q_{t}^{k}\left(y_{t}^{k}\right)\right.\right. \\
& \left.\left.+\sum_{i=1}^{2} H_{i t}^{k}\left(I_{i t}^{k}\right)\right]\right]
\end{aligned}
$$

subject to

$$
\begin{gathered}
I_{1 t}^{k}=I_{1, t-1}^{k}+x_{t}^{k}-r_{1 t}^{k}, k=1,2, t=1,2, \ldots, T \\
I_{2 t}^{k}=I_{2, t-1}^{k}+x_{t}^{k}+y_{t}^{j}-y_{t}^{k}-r_{2 t}^{k}, \\
j, k=1,2, j \neq k, t=1,2, \ldots, T \\
I_{i 0}^{k}=I_{i T}^{k}=0, i, k=1,2 \\
x_{t}^{k} \geq 0, y_{t}^{k} \geq 0, I_{i t}^{k} \geq 0, i, k=1,2, t=1,2, \ldots, T
\end{gathered}
$$

The objective function $F(z)$ is concave, since $P_{t}^{k}(\bullet)$, $Q_{t}^{k}(\bullet)$, and $H_{i t}^{k}(\bullet)$ are concave functions. The constraints (1)-(4) of the problem (PB) define a closed bounded convex set. Since $F(z)$ is non-decreasing concave, there exists an extreme point optimal solution. The constraints (1)-(4) can be described as a single source network problem. The network is shown in Figure 1.

The constraints (1) - (4) can be shown to be totally unimodular as done in [15]. Therefore, since the demands are integers, any extreme point solution consists of integer values 
for all variables.

In the next section, we shall describe the properties of an optimal solution and an algorithm for finding the solution.

\section{Extreme Flows and Optimal Schedules}

\subsection{Characteristics of an Optimal Solution}

Since the constraints (1) - (4) define a convex set, and $F(\bullet)$ is concave, the minimum cost is attained at an extreme point of the set. So we shall concentrate on finding such an extreme point solution.

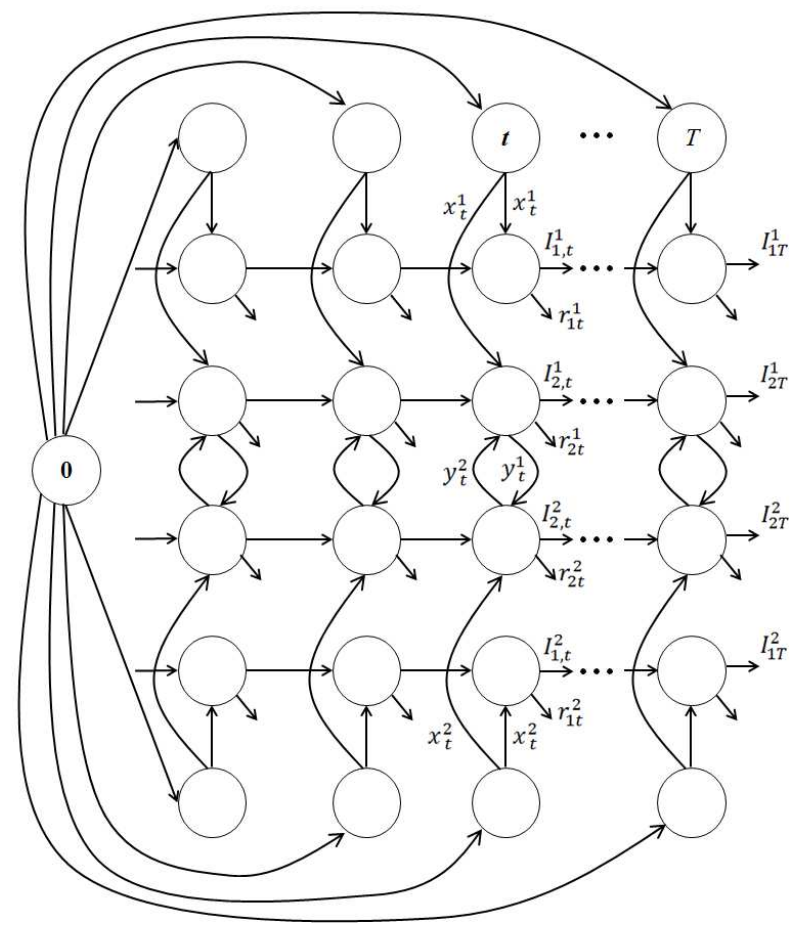

Figure 1. A Network Representation

We shall first derive certain optimality properties, some of which will be used later to construct a solution algorithm. As done in [16], it can be shown easily that a feasible flow in the network of Figure 1 corresponds to an extreme flow if and only if it does not contain any cycle with positive flows. Furthermore, referring to [2], an extreme flow is composed of some nodes and arcs, where each of the nodes has at most one positive input. These extreme flow properties lead to the following sufficient conditions for an extreme point solution of the given problem:

(i) $I_{1, t-1}^{k} \bullet I_{2, t-1}^{k} \bullet x_{t}^{k}=0, k=1,2$ and $t=1,2, \ldots, T$

(ii) $y_{t}^{k} \cdot I_{2, t-1}^{k}=0, k=1,2$ and $t=1,2, \ldots, T$

(iii) $x_{t}^{k} \bullet y_{t}^{j}=0, k, j=1,2$ and $k \neq j, t=1,2, \ldots, T$

However, the extreme flow property clarified in [2] is for single source networks, so that it is not always satisfied in our model. Therefore, we shall find some additional properties that are useful for our algorithm development.

Let period $t$ be called an "inventory point" if $I_{i t}^{k}=0$ for at least one of both $i$ and $k$, that is, $\prod_{i, k=1,2} I_{i t}^{k}=0$. And a production (transportation) point of a schedule $z$ in region $k$ is defined as a period $t$ in which $x_{t}^{k}>0\left(y_{t}^{k}>0\right)$.

Theorem 1 Let $u$ and $v$ be two consecutive inventory points $(u<v)$. If $z$ is an extreme point solution, then the following properties are satisfied:

(i) In each region, there exists at most one production point between $u$ and $v$ (including $v$ )

(ii) $y_{t_{1}}^{1} \cdot y_{t_{2}}^{2}=0, u<t_{1}, t_{2} \leq v$

(iii) $y_{t_{3}}^{k} \cdot y_{t_{4}}^{k}=0, k=1,2, u<t_{3}, t_{4} \leq v$

Proof of $(i)$. We consider a region $k$. Assume that a feasible solution $z, \quad z=\left(x_{1}^{1}, \ldots, x_{T}^{1} ; x_{1}^{2}, \ldots, x_{T}^{2} ; y_{1}^{1}, \ldots, y_{T}^{1}\right.$; $y_{1}^{2}, \ldots, y_{T}^{2}$ ) satisfies (ii) and (iii), but not (i); that is, there exists two distinct production points $t_{1}$ and $t_{2}$ such that $I_{1, t-1}^{k} \bullet I_{2, t-1}^{k} \bullet x_{t}^{k} \neq 0$ for $t=t_{1}, t_{2}\left(u<t_{1}<t_{2} \leq v\right)$. Then there exists $\delta>0$ such that $\delta=(1 / 2) \min \left\{x_{t_{1}}^{k}, x_{t_{2}}^{k}, I_{i t}^{k}, t_{1} \leq t<t_{2}, i=1,2\right\}$. Therefore, the following two solutions $\bar{z}$ and $\hat{z}$ also satisfy (ii) and (iii):

a) $\bar{x}_{t_{1}}^{k}=x_{t_{1}}^{k}-\delta, \bar{x}_{t_{2}}^{k}=x_{t_{2}}^{k}+\delta$ and for all other $t$, $\bar{x}_{t}^{j}=x_{t}^{j}, \bar{y}_{t}^{j}=y_{t}^{j}, j=1,2$

b) $\hat{x}_{t_{1}}^{k}=x_{t_{1}}^{k}+\delta, \hat{x}_{t_{2}}^{k}=x_{t_{2}}^{k}-\delta$ and for all other $\mathrm{t}$, $\hat{x}_{t}^{j}=x_{t}^{j}, \hat{y}_{t}^{j}=y_{t}^{j}, j=1,2$

Since $\delta>0$, the above solutions are feasible. However, $x_{t_{1}}^{k}=\frac{1}{2}\left(\bar{x}_{t_{1}}^{k}+\hat{x}_{t_{1}}^{k}\right)$ and $x_{t_{2}}^{k}=\frac{1}{2}\left(\bar{x}_{t_{2}}^{k}+\hat{x}_{t_{2}}^{k}\right)$. Hence $z=$ $\frac{1}{2}(\bar{z}+\hat{z}) \quad, \quad$ where $\quad \bar{z}=\left(\bar{x}_{1}^{1}, \ldots, \bar{x}_{T}^{1} ; \bar{x}_{1}^{2}, \ldots, \bar{x}_{T}^{2}\right.$; $\left.\bar{y}_{1}^{1}, \ldots, \bar{y}_{T}^{1} ; \bar{y}_{1}^{2}, \ldots, \bar{y}_{T}^{2}\right) \quad, \quad \hat{z}=\left(\hat{x}_{1}^{1}, \ldots, \hat{x}_{T}^{1} ; \hat{x}_{1}^{2}, \ldots, \hat{x}_{T}^{2}\right.$; $\left.\hat{y}_{1}^{1}, \ldots, \hat{y}_{T}^{1} ; \hat{y}_{1}^{2}, \ldots, \hat{y}_{T}^{2}\right)$. This implies that $z$ is not an extreme point. It completes the proof of (i).

Proof of (ii). Likewise, assume $\bar{y}_{1}^{1}, \ldots, \bar{y}_{T}^{1}$; that a feasible solution $z$ satisfies (i) and (iii), but not (ii). Let $t_{1}$ and $t_{2}$ be two distinct positive transportation points; that is, $y_{t_{1}}^{1}>$ 0 and $y_{t_{2}}^{2}>0$, where $u<t_{1} \leq t_{2} \leq v$. Then we can find a real value $\delta>0$ such that

$$
\delta=\frac{1}{2} \min \left\{y_{t_{1}}^{1}, y_{t_{2}}^{2}, I_{2 t}^{k}, t_{1} \leq t<t_{2}, k=1,2\right\} .
$$

Following the procedures described for the proof of (i), we can show that $z$ is not an extreme point. Similarly, (iii) can be proved. Thus, the proof is completed by contraposition.

\subsection{Dynamic Programming Approach}

We can solve the problem (PB) by applying a dynamic programming approach. The approach is based on using the vector $I_{t}=\left(I_{1 t}^{1}, I_{2 t}^{1} ; I_{1 t}^{2}, I_{2 t}^{2}\right)$ that satisfies the definition of "inventory point", which represents the state variables.

From the totally unimodular property, it follows that an extreme point consists of integer values. Hence, a search for an optimal solution is limited to integer values of $I_{i t}^{k}$ that satisfy the conditions, $0 \leq I_{1 t}^{k} \leq R_{1}^{k}(t+1)$ and $0 \leq$ $\sum_{k=1}^{2} I_{2 t}^{k} \leq \sum_{k=1}^{2} R_{2}^{k}(t+1)$. Therefore, our goal is to find an optimal sequence of successive inventory points and the associated solutions of the sequence. The minimum cost incurred between any two consecutive inventory points can be computed using the aforementioned properties for extreme points.

We now describe the dynamic programming approach to solve the problem (PB). Let $d_{u v}\left(I_{u}, I_{v}\right)$ be the minimal cost between two successive inventory points $u$ and $v$. Then, 
$d_{u v}\left(I_{u}, I_{v}\right)=$

$\min _{x_{t}^{k}, y_{t}^{k}} \sum_{t=u+1}^{v}\left[\sum_{k=1}^{2} P_{t}^{k}\left(x_{t}^{k}\right)+Q_{t}^{k}\left(y_{t}^{k}\right)+\sum_{i=1}^{2} H_{i t}^{k}\left(I_{i t}^{k}\right)\right](5)$

subject to

(i) $I_{u}$ and $I_{v}$ are inventory point values,

(ii) Constraints (1) - (4) are satisfied for $t=u+1, \ldots, v$,

(iii) $\prod_{i, k=1,2} I_{i t}^{k}>0, t=u+1, \ldots, v-1$,

(iv) $x_{t}^{k}$ and $y_{t}^{k}$ satisfy the properties described in Theorem 1, for all $t$ and $k,(t=u+1, \ldots, v ; k=$ $1,2)$.

Since all the possible sequence of those $(u-v)$ subproblems include all the extreme points of the constraints (1) - (4), there exists an optimal solution of (PB) that consists of a sequence of subproblems with $d_{u v}\left(I_{u}, I_{v}\right)$ values as defined above. The computation of $d_{u v}\left(I_{u}, I_{v}\right)$ values will be described in detail in the next section.

Furthermore, let $f_{t}\left(I_{t}\right)$ be the cost of optimal policy over periods $t+1, \ldots, T$, given that period $t$ is an inventory point with a value $I_{t}$. Given $d_{u v}\left(I_{u}, I_{v}\right)$ values for all $u$ and $v$, the following dynamic programming formulation holds:

$$
\begin{gathered}
f_{u}\left(I_{u}\right)=\min _{u+1 \leq v \leq T}\left[d_{u v}\left(I_{u}, I_{v}\right)+f_{v}\left(I_{v}\right)\right], I_{u} \in G_{u}, \\
u=T-1, \ldots, 1,0 \\
f_{T}\left(I_{T}\right)=0, I_{0}=(0,0 ; 0,0) \text { and } I_{T}=(0,0 ; 0,0),
\end{gathered}
$$

where $G_{u}$ is the set of all possible "inventory point" values at period $u$. The equations (6) and (7) indicate a backward dynamic procedure starting $u=T-1$ and ending $u=0$. $f_{0}\left(I_{0}\right)$ represents the cost of an optimal solution.

Most of the computational efforts involved in solving the problem (PB) is spent on computing $d_{u v}\left(I_{u}, I_{v}\right)$ values for each $(u-v)$ subproblem solution. Thus we will concentrate our efforts on solving more efficiently such subproblems.

\subsection{Approach Solution Procedure of a (u-v) Subproblem}

Let $D_{i}^{k}$ be the production amount of product $i$ at facility $k$ (i.e., region $k$ ), during periods $u+1, \ldots, v$, given $I_{u}$ and $I_{v}$ as successive inventory points. Then,

$$
\begin{gathered}
D_{1}^{k}=I_{1 v}^{k}-I_{1 u}^{k}+\sum_{t=u+1}^{v} r_{1 t}^{k}, k=1,2 \\
D_{2}^{k}=I_{2 v}^{k}-I_{2 u}^{k}+\sum_{t=u+1}^{v} r_{2 t}^{k}+\sum_{t=u+1}^{v}\left(y_{t}^{k}-y_{t}^{j}\right), \\
\quad k, j=1,2,(k \neq j)
\end{gathered}
$$

From the assumption that $x_{1 t}^{k}=x_{2 t}^{k}$, for all $t$, we see that $D^{k}=D_{1}^{k}=D_{2}^{k}, k=1,2$. And let $Y^{k}$ be the transportation amount from region $k$ to region $j(k, j=1,2, k \neq j)$ over periods $u+1, \ldots, v$; that is, $Y^{k}=\sum_{t=u+1}^{v} y_{t}^{k}$. Then equation (9) can be expressed as follows:

$$
D_{2}^{k}=I_{2 v}^{k}-I_{2 u}^{k}+\sum_{t=u+1}^{v} r_{2 t}^{k}+Y^{k}-Y^{j}
$$

Since $D_{1}^{k}=D_{2}^{k}, k=1,2$, we can see from (9') that

$$
\begin{gathered}
Y^{1}-Y^{2}=\left(I_{1 v}^{1}-I_{1 u}^{1}+\sum_{t=u+1}^{v} r_{1 t}^{1}\right)-\left(I_{2 v}^{1}-I_{2 u}^{1}\right. \\
\left.+\sum_{t=u+1}^{v} r_{2 t}^{1}\right) \\
Y^{1}-Y^{2}=\left(\begin{array}{c}
(10) \\
\left(I_{2 v}^{2}-I_{2 u}^{2}+\sum_{t=u+1}^{v} r_{2 t}^{2}\right)-\left(I_{1 v}^{2}-I_{1 u}^{2}\right. \\
\left.+\sum_{t=u+1}^{v} r_{1 t}^{2}\right)
\end{array}\right.
\end{gathered}
$$

Subsequently, if the right hand sides of the equation (10) and (11) are not equal, then the subproblem is infeasible. Otherwise the subproblem is feasible. Letting $Y=Y^{1}-Y^{2}$ and $|Y|$ denote the absolute value of $Y$, we can see from Theorem 1 that either $Y^{1}=|Y|$ or $Y^{2}=|Y|$. In other words, if $Y>0, Y=Y^{1}$ but $Y<0,-Y=Y^{2}$. Further, if any one of $D^{1}$ and $D^{2}$ is negative, then the subproblem is also infeasible.

Moreover, suppose that $I_{1 u}^{k} \neq 0$ but $I_{2 u}^{k}=0$ and $Y^{j}=$ $0(k \neq j, k, j=1,2)$. Then, $y_{u+1}^{j}>0$ or $x_{u+1}^{k}=D^{k}$ is required to satisfy the demand $r_{2, u+1}^{k}$. However, since $Y^{j}=0$, it is required that $x_{u+1}^{k}=D^{k}$. This implies that in this case, the production point in region $k$ is fixed at period $u+1$.

These properties lead to the following proposition, which describes the feasible location of a transportation point for a $(u-v)$ subproblem.

Proposition 1 Assume that a $(u-v)$ subproblem is given and $Y^{j}>0$. Then the optimal transportation point within the subproblem can be only one point between period $u$ and $\hat{t}$ (including $\hat{t}$ ), where period $t$ satisfies the following relationships:

(i) If $0<Y^{j}<I_{2 v}^{k}+r_{2 v}^{k}$, for $k, j=1,2$ and $k \neq j$, then $\hat{t}=v$

(ii) If $I_{2 v}^{k}+r_{2 v}^{k}<Y^{j}<I_{2 v}^{k}+\sum_{t=u+1}^{v} r_{2 t}^{k}$, for $k, j=1,2$ and $k \neq j$, then there exists a period $\hat{t}$ such that $I_{2 v}^{k}+\sum_{t=\hat{t}+1}^{v} r_{2 t}^{k}<Y^{j}<I_{2 v}^{k}+\sum_{t=\hat{t}}^{v} r_{2 t}^{k}, u+1 \leq \hat{t}<$ $v$

(iii) If $Y^{j}=I_{2 v}^{k}+\sum_{t=u+1}^{v} r_{2 t}^{k}$, for $k, j=1,2$ and $k \neq j$, then $\hat{t}=u+1$

Proof. Since proofs of (i) and (iii) are obvious, we consider only the proof of (ii). Consider period $t^{*}\left(u+1 \leq \hat{t}<t^{*} \leq\right.$ $v)$ such that $y_{t^{*}}^{j}>0$ so that $y_{t^{*}}^{j}=Y^{j}(>0)$ from Theorem 1 . Then, from the hypothesis in (ii), we can show that

$$
I_{2 v}^{k}+\sum_{t=t^{*}}^{v} r_{2 t}^{k}<y_{t^{*}}^{j}
$$

Suppose that if $I_{2 v}^{k}+\sum_{t=t^{*}}^{v} r_{2 t}^{k}<y_{t^{*}}^{j}$. Then, since the inventory amount at the end of period $v$ is greater than the given inventory $I_{2 v}^{k}$, its solution violates the definition of $(u-v)$ subproblem. And if $I_{2 v}^{k}+\sum_{t=t^{*}}^{v} r_{2 t}^{k}=y_{t^{*}}^{j}$, then it indicates that $I_{2, t^{*}-1}^{k}=0$, which is not allowed in a $(u-v)$ subproblem, either. Therefore, the transportation point must not be located after period $\hat{t}$. It completes the proof.

Transportation policies are summarized in the following Theorem.

Theorem 2 Suppose that $Y^{j}-Y^{k}>0$, for $j, k=1,2$ and $j \neq k$. Then, we can use the following policies to get the optimal transportation point.

(i) If $I_{2 u}^{k}+D^{k}<r_{2 u}^{k}$, then $y_{u+1}^{j}=Y^{j}$ and all other transportation variables have values of zero

(ii) Otherwise, there exists only one transportation point $p$ between $u$ and $\hat{t}$ (including $\hat{t}$ ) such that $y_{p}^{j}=Y^{j}$, where $\hat{t}$ is the period obtained from Proposition 1 .

Likewise, for the case of $I_{1 u}^{k}>0,(k=1,2)$, a similar relationship can be constructed for the production point.

Proposition 2 Consider the case of $I_{1 u}^{k}>0,(k=1,2)$. Let 
$t_{k}^{\prime}$ and $t_{k}^{\prime \prime}\left(u<t_{k}^{\prime}, t_{k}^{\prime \prime} \leq v\right)$ be the periods in which the following relationships are satisfied:

(i) If $I_{1 u}^{k} \leq r_{1, u+1}^{k}$, then $t_{k}^{\prime}=u+1$

(ii) If $r_{1, u+1}^{k}<I_{1 u}^{k}<\sum_{t=u+1}^{v} r_{1 t}^{k}$, then there exists $t_{k}^{\prime}$ such that $\sum_{t=u+1}^{t_{k}^{\prime}-1} r_{1 t}^{k} \leq I_{1 u}^{k}<\sum_{t=u+1}^{t_{k}^{\prime}} r_{1 t}^{k}$, for $u+1<t_{k}^{\prime} \leq v$

(iii) If $I_{1 u}^{k} \geq \sum_{t=u+1}^{v} r_{1 t}^{k}$, then $t_{k}^{\prime}=v$

(iv) If $D^{k}<I_{1 v}^{k}+r_{1 v}^{k}$, then $t_{k}^{\prime \prime}=v$

(v) If $D^{k} \geq I_{1 v}^{k}+r_{1 v}^{k}$, then there exists $t_{k}^{\prime \prime}$ such that $I_{1 v}^{k}+\sum_{t=t_{k}^{\prime \prime}+1}^{v} r_{1 t}^{k} \leq D^{k}<I_{1 v}^{k}+\sum_{t=t_{k}^{\prime \prime}}^{v} r_{1 t}^{k} \quad, \quad$ for $u+1 \leq t_{k}^{\prime \prime}<v$

Then, there exists only one optimal production point located between $u$ and $t_{k}$ (including $t_{k}$ ) in region $k$, where $t_{k}=\min \left\{t_{k}^{\prime}, t_{k}^{\prime \prime}\right\}$.

Proof. If $t_{k}=v$, then the above property is obviously satisfied. Consider the case of $u<t_{k}<v$.

Suppose that $t_{k}=t_{k}^{\prime}$ and in period $\hat{t}\left(u<t_{k}^{\prime}<\hat{t} \leq v\right)$, $x_{\hat{t}}^{k}>0$ (i.e., $x_{\hat{t}}^{k}=D^{k}>0$ ). For the case of (i), it leads to an infeasible solution for the $(u-v)$ subproblem, since $I_{1, u+1}^{k} \leq 0$.

Next, consider the case (ii). In this case, $I_{1 u}^{k}-\sum_{t=u+1}^{t_{k}} r_{1 t}^{k}<$ 0 . Hence, the amount of $D^{k}$ produced at period $\hat{t}$ incurs negative inventories $I_{1 t}^{k}<0$, at each period $t, t=t_{k}^{\prime}, \ldots, \hat{t}-$ 1. Therefore, its solution is infeasible.

Furthermore, in the case of which $t_{k}=t_{k}^{\prime \prime}$, it is similarly proved by following the proof steps in Proposition 1.

These production policies are summarized in Table 1.

Table 1. Production Policies

\begin{tabular}{|c|c|c|}
\hline$I_{2 u}^{k} I_{1 u}^{k}$ & 0 & positive \\
\hline 0 & $x_{u+1}^{k}=D^{k}$ & $x_{t}^{k}=D^{k}, u<t \leq t_{k}$ \\
\hline positive & $x_{u+1}^{k}=D^{k}$ & $x_{t}^{k}=D^{k}, u<t \leq t_{k}$ \\
\hline
\end{tabular}

Theorem 1, Theorem 2, and the information in Table 1 can be employed together to solve every $(u-v)$ subproblem, which represent out solution algorithm.

In the next section, a numerical example is presented to illustrate the algorithm.

\section{A Numerical Example}

We illustrate the algorithm with a 3-period two-product two-facility problem. The production, inventory and transportation cost functions are given as follows:

$P_{t}^{1}\left(x_{t}^{1}\right)=\left(30+8 x_{t}^{1}\right)(0.9)^{t-1}$

$P_{t}^{2}\left(x_{t}^{2}\right)=\left(20+10 x_{t}^{2}\right)(0.9)^{t-1}$

$H_{i t}^{k}\left(I_{i t}^{k}\right)=5 I_{i t}^{k}(0.9)^{t-1}$

$Q_{t}^{k}\left(y_{t}^{k}\right)=5 y_{t}^{k}(0.9)^{t-1}$

$t=1,2,3$ and $k=1,2$

The demand data for $\left\{r_{1 t}^{1}\right\},\left\{r_{2 t}^{1}\right\},\left\{r_{1 t}^{2}\right\}$ and $\left\{r_{2 t}^{2}\right\}$ are given as $(2,2,1),(1,1,1),(1,1,1)$ and $(1,2,2)$, respectively.
Conveniently, we shall assume that $y_{t}^{2}=0, t=1,2, \ldots, T$. We know that the problem can be decomposed into several $(u-v)$ subproblems, each of which can be solved independently by use of Theorem 1 and the relations (5), (6) and (7).

Let $S_{t i}$ be the $i^{t h}$ state variable at inventory point $t$. Then the recursive equations shown in (6) and (7) are represented as follows:

$$
\begin{gathered}
f_{u}\left(S_{u i}\right)=\min _{u+1 \leq v \leq T}\left[d_{u v}\left(S_{u i}, S_{v j}\right)+f_{v}\left(S_{v j}\right)\right], S_{u i} \in G_{u}, \\
u=T-1, \ldots, 1,0, i=1, \ldots, N_{u}, j=1, \ldots, N_{v} \\
f_{T}\left(S_{T 1}\right)=0, S_{T 1}=I_{T}=(0,0 ; 0,0), \\
S_{01}=I_{0}=(0,0 ; 0,0)
\end{gathered}
$$

Where $G_{u}$ is the set of all possible "inventory point" values at period $u$ and $N_{t}$ is the number of states at period $t . S_{t i}$ values are shown in Table 2.

Table 2. $S_{t i}$ values

\begin{tabular}{ccc}
\hline $\boldsymbol{i}$ & $\boldsymbol{S}_{\mathbf{1 i}}$ & $\boldsymbol{S}_{\mathbf{2 i}}$ \\
\hline 1 & $(0,0 ; 1,0)$ & $(0,0 ; 1,0)$ \\
2 & $(0,0 ; 2,1)$ & $(0,0 ; 2,1)$ \\
3 & $(0,0 ; 3,2)$ & $(0,1 ; 0,0)$ \\
4 & $(0,1 ; 0,0)$ & $(0,1 ; 1,1)$ \\
5 & $(0,1 ; 1,1)$ & $(0,2 ; 0,1)$ \\
6 & $(0,1 ; 2,2)$ & $(1,1 ; 1,0)$ \\
7 & $(1,0 ; 2,0)$ & $(1,2 ; 0,0)$ \\
8 & $(1,0 ; 3,1)$ & $(1,3 ; 0,1)$ \\
9 & $(1,0 ; 4,2)$ & - \\
10 & $(2,1 ; 2,0)$ & - \\
11 & $(2,2 ; 1,0)$ & - \\
12 & $(2,3 ; 0,0)$ & - \\
13 & $(3,2 ; 2,0)$ & - \\
14 & $(3,3 ; 1,0)$ & \\
15 & $(3,4 ; 0,0)$ & \\
\hline
\end{tabular}

Consider the subproblem $d_{03}\left(S_{01}, S_{31}\right)$, where $S_{01}=S_{31}=$ $(0,0 ; 0,0)$. By equations (8) and $(11), D^{1}=5, D^{2}=3$, and $Y^{1}=2$. According to the information in Theorem 2 and Table 1 , policy (i) yields $x_{1}^{1}=5, x_{1}^{2}=3, y_{1}^{1}=2$ with the total cost of 207.5, policy (ii) yields $x_{1}^{1}=5, x_{1}^{2}=3, y_{2}^{1}=2$ and other variables are zero with a total cost 206.5. Hence the result of the policy (ii) is the optimal one. As another subproblem, the values of $d_{23}\left(S_{2 i}, S_{31}\right)$ are shown in Table 3. Other $d_{u v}\left(S_{u i}, S_{v j}\right)$ values for $0 \leq u<v \leq 3$ can be calculated similarly.

After solving all such subproblems, the shortest path is searched using the dynamic programming formulation in equations $\left(6^{\prime}\right)$ and $\left(7^{\prime}\right)$. The $f_{t}\left(S_{t i}\right)$ values can be calculated and its results are shown in Table 4.

Table 3. (2-3) subproblem

\begin{tabular}{cccc}
\hline $\boldsymbol{i}$ & $\boldsymbol{S}_{\mathbf{2 i}}$ & $\boldsymbol{S}_{\mathbf{3 1}}$ & $\boldsymbol{d}_{\mathbf{2 3}}\left(\boldsymbol{S}_{\mathbf{2 i}}, \boldsymbol{S}_{\mathbf{3 1}}\right)$ \\
\hline 1 & $(0,0 ; 1,0)$ & $(0,0 ; 0,0)$ & 55.08 \\
2 & $(0,0 ; 2,1)$ & $(0,0 ; 0,0)$ & 30.78 \\
3 & $(0,1 ; 0,0)$ & $(0,0 ; 0,0)$ & 59.13 \\
\hline
\end{tabular}




\begin{tabular}{cccc}
\hline $\boldsymbol{i}$ & $\boldsymbol{S}_{\mathbf{2 i}}$ & $\boldsymbol{S}_{\mathbf{3 1}}$ & $\boldsymbol{d}_{\mathbf{2 3}}\left(\boldsymbol{S}_{\mathbf{2 i}}, \boldsymbol{S}_{\mathbf{3 1}}\right)$ \\
\hline 4 & $(0,1 ; 1,1)$ & $(0,0 ; 0,0)$ & 34.83 \\
5 & $(0,2 ; 0,1)$ & $(0,0 ; 0,0)$ & 38.88 \\
6 & $(1,1 ; 1,0)$ & $(0,0 ; 0,0)$ & 24.30 \\
7 & $(1,2 ; 0,0)$ & $(0,0 ; 0,0)$ & 28.35 \\
8 & $(1,3 ; 0,1)$ & $(0,0 ; 0,0)$ & 8.10 \\
\hline
\end{tabular}

Table 4. $f_{t}\left(S_{t i}\right)$ values

\begin{tabular}{ccc}
\hline$i$ & $\boldsymbol{f}_{\mathbf{1}}\left(\boldsymbol{S}_{\mathbf{1 i}}\right)$ & $\boldsymbol{f}_{\mathbf{2}}\left(\boldsymbol{S}_{\mathbf{2}}\right)$ \\
\hline 1 & 111.15 & 55.08 \\
2 & 90.45 & 30.78 \\
3 & 75.15 & 59.13 \\
4 & 115.20 & 34.83 \\
5 & 94.95 & 38.88 \\
6 & 79.20 & 24.30 \\
7 & 106.20 & 28.35 \\
8 & 79.20 & 8.10 \\
9 & 78.48 & - \\
10 & 80.28 & - \\
11 & 84.33 & - \\
12 & 63.90 & - \\
13 & 58.50 & - \\
14 & 62.55 & - \\
15 & 66.60 & - \\
\hline
\end{tabular}

$$
f_{0}\left(S_{01}\right)=195.5
$$

The optimal policy of the entire problem is then $x_{1}^{1}=2$, $x_{2}^{1}=3, x_{3}^{1}=0, x_{1}^{2}=2, x_{2}^{2}=0, x_{3}^{2}=1, y_{1}^{1}=0, y_{2}^{1}=1$, and $y_{3}^{1}=1$ with the total minimum cost 195.95. Its optimal sequence is $S_{01} \rightarrow S_{15} \rightarrow S_{27} \rightarrow S_{31}$.

\section{Conclusion}

In this paper, we described a two-product two-region production and transportation model. Using a network flow approach, properties of extreme points were identified. Then, they were used to develop an efficient solution search procedure for each independent subproblem, based upon which a dynamic programming algorithm was constructed for an optimal solution search. However, the computational efficiency of the algorithm is closely dependent on the number of nodes associated with the demand size in each period as well as the planning horizon $T$. Thus, our attention is paid to further research effort on decreasing the number of such nodes for a better algorithm development. And also the model can be extended to the case in which capacity bounds on transportation are allowed.

\section{References}

[1] H. M. Wagner and T. M. Whitin, "Dynamic Version of the Economic Lot Size Model”, Management Science, vol. 14, pp.429-450, 1968.

[2] W. I. Zangwill, "Minimum Concave Cost Flows in Certain Network", Management Science, vol. 14, pp.429-450, 1968.
[3] M. Florian and M. Klein, "Deterministic Production Planning with Concave Costs and Capacity Constraints", Management Science, vol. 18, pp.12-20, 1971.

[4] C. S. Sung, "A Production Planning Model for Multi-Product Facilities" Journal of the Operations Research Society of Japan, vol. 28, no. 4, pp.345-358, 1985.

[5] H. Luss, "A Capacity-Expansion Model for Two Facility Types", Naval Research Logistics Quarterly, vol. 26, pp.291-303, 1979.

[6] Nafee Rizk and Alain Martel, "Supply Chain Flow Planning Methods: A Review of the Lot-Sizing Literature", Working Paper DT-2001-AM-1, Centre de recherche sur les technologies de l'organisation réseau (CENTOR), Université Laval, QC, Canada, January 2001.

[7] B. Karimi, S.M.T. Fatemi Ghomi, and J.M. Wilson, "The capacitated lot sizing problem: a review of models and algorithms", The International Journal of Management Science, Omega 31, pp.365-378, 2003.

[8] Lisbeth Buschkuhl, Florian Sahling, Stefan Helber, and Horst Tempelmeier, "Dynamic capacitated lot-sizing problems: a classification and review of solution approaches", OR Spectrum, vol. 32, pp.231-261, 2010.

[9] A. Clark, B. Almada-Lobo, and C. Almeder, "Lot sizing and scheduling: industrial extensions and research opportunities", International Journal of Production Research, vol. 49, pp. 2457 $2461,2011$.

[10] Endy Suwondo and Henry Yuliando, "Dynamic Lot-Sizing Problems: A Review on Model and Efficient Algorithm", Agroindustrial Journal, vol. 1, issue 1, pp. 36-49, 2012.

[11] R. K. Oliver and M. D. Webber, "Supply-Chain Management: Logistics Catches up with Strategy", in Christopher, M. Logistics: The Strategic Issues, Chapman Hall, London, pp. 63-75, ISBN 0-412-41550-X, 1992.

[12] Adulyasak, Yossiri, Jean-Francois Cordeau, and Raf Jans, "The Production Routing Problem: A Review of Formulations and Solution Algorithms", Computers \& Operations Research, available online 7 February 2014.

[13] L.C. Coelho, J.-F. Cordeau, G. Laporte, "The inventory-routing problem with transshipment", Computers and Operations Research, vol. 39, pp. 2537-2548, 2012.

[14] D. Ozdemir, E. Yucesan, Y. T. Herer, "Multi-location transshipment problem with capacitated production", European Journal of Operational Research, vol. 226, pp. 425-435, 2013.

[15] A. J. Hoffman and J. B. Kruskal, "Integral Boundary Points of Convex Polyhedra", in H. W. Tucker (eds.) Linear Inequalities and Related Systems, Annals of Mathematics Study, no. 38, Princeton Univ. Press, Princeton, New Jersey, pp.233-246, 1956.

[16] G. B. Danzig, "Linear Programming and Extensions", Princeton Univ. Press, Princeton, N. J., 1963. 